\title{
EL NO POSITIVISMO INCLUYENTE *
}

Robert Alexy

Universidad de Kiel

RESUMEN. Partiendo de la distinción entre las dos formas de positivismo y las tres de no-positivismo, sostengo que sólo uno de estos cinco conceptos de derecho es defendible: el no-positivismo incluyente. En el centro de mi argumentación está la tesis de la corrección, la cual establece que el derecho necesariamente formula una pretensión de corrección. Esta tesis implica a su vez la tesis de la naturaleza dual, según la cual el derecho comprende tanto una dimensión real o autoritativa como una dimensión ideal o crítica. La idea de la naturaleza dual del derecho es el fundamento de la fórmula de Radbruch. Ésta señala, en su formulación más simple, que la injusticia extrema no es derecho.

Palabras clave: positivismo, no-positivismo, no-positivismo incluyente, tesis de la corrección, fórmula de Radbruch.

\section{Inclusive Non-Positivism}

ABSTRACT. On the basis of the distinction between two forms of positivism and three forms of nonpositivism, I argue that only one of these five concepts of law is defensible: inclusive non-positivism. The basis of my argument is the correctness thesis, which says that law necessarily makes a claim to correctness. The doctrine of correctness implies the dual nature thesis, which says that law comprises a real or authoritative dimension as well as an ideal or critical dimension. The dual nature of law is the basis of the Radbruch formula. It says, in its shortest form, that extreme injustice is not law.

Keywords: positivism, non-positivism, inclusive non-positivism, correctness thesis, Radbruch formula.

* Fecha de recepción: 20 de septiembre de 2013. Fecha de aceptación: 31 de octubre de 2013. 


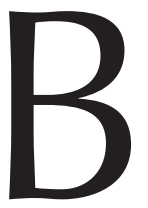

ajo el rótulo general del concepto y la naturaleza del derecho, uno de los problemas fundamentales es la relación entre el derecho y la moral. En este contexto dos tendencias han competido por el dominio teórico por más de dos milenios: el positivismo y el no-positivismo.

\section{TESIS DE LA SEPARACIÓN Y TESIS DE LA CONEXIÓN}

Todos los positivistas defienden la tesis de la separación. En su forma más general, ésta dice que no hay una conexión necesaria entre el derecho tal como es y el derecho como debe ser. $\mathrm{O}$, en una formulación más precisa, afirma que no hay una conexión necesaria entre la validez jurídica o corrección jurídica, por un lado, y el mérito moral o la corrección moral por el otro. Por el contrario, todos los no positivistas defienden la tesis de la conexión, según la cual hay una vinculación necesaria entre la validez jurídica o corrección jurídica, por un lado, y el mérito moral o corrección moral, por el otro (ALEXY, 2008b, 284-285). Esto implica que, con el fin de determinar el concepto y la naturaleza del derecho, todas las teorías positivistas están limitadas a dos elementos, específicamente, a la expedición autoritativa y a la eficacia social. La caracterización de las teorías no positivistas, en cambio, incluye un tercer elemento: la corrección del contenido (ALEXY, 2002a, 3-4).

\section{LAS FORMAS DE POSITIVISMO Y NO-POSITIVISMO}

¿Cuál tesis es más defendible? ¿La de la separación o la de la conexión? Ambas se prestan a una variedad de interpretaciones. La respuesta a nuestra pregunta principal gira en torno a esas diversas interpretaciones.

Dentro del positivismo, la distinción entre el positivismo jurídico incluyente y excluyente es la diferencia más importante en lo que respecta a la relación entre el derecho y la moral. El positivismo excluyente, que ha defendido prominentemente J. RAZ, sostiene que la moral está necesariamente excluida del concepto de derecho (RAZ, 2009, 47). El positivismo incluyente, defendido, por ejemplo, por J. ColEman, dice que la moral no está ni necesariamente excluida ni necesariamente incluida. La inclusión se considera entonces como una cuestión contingente o convencional dependiendo de lo que el derecho positivo, de hecho, establece (ColEman, 1996, 316). Esto trae implícito que la relación entre el derecho y la moral, tanto en el positivismo incluyente como en el positivismo excluyente, está determinada únicamente por lo que es expedido autoritativamente y es socialmente eficaz, es decir, por hechos sociales. El positivismo incluyente es una forma de positivismo, porque afirma que la decisión inicial, en un sistema jurídico particular, de incluir la moralidad en el derecho es contingente o convencional (ALEXY, 2012, 4). El no-positivismo sostiene no sólo, en contra del positivismo excluyente, que la moralidad no está necesariamente excluida, sino que también se sitúa en contra del positivismo incluyente al señalar que está necesariamente incluida, por lo tanto el no-positivismo es contrario a ambas formas de positivismo. 
Las diferencias dentro del no-positivismo no son menos importantes que las diferencias dentro del positivismo. De especial importancia para el debate sobre el concepto y la naturaleza del derecho son las diferencias que surgen de los diferentes efectos que en la validez jurídica son atribuibles a los defectos morales. El no-positivismo puede determinar el efecto sobre la validez jurídica que se deriva de los defectos morales de tres maneras diferentes. Podría darse la circunstancia de que la validez jurídica se pierda en todos los casos, o que la validez jurídica se pierda en algunos casos y en otros no, o por último, que la validez jurídica no se vea afectada de ninguna manera en absoluto (ALEXY, 2008b, 287).

La primera posición, de acuerdo con la cual todo defecto moral produce invalidez jurídica, es la versión más radical del no-positivismo. Esta posición puede ser caracterizada como «no-positivismo excluyente» a fin de expresar la idea de que todos y cada uno de los defectos morales excluyen la validez jurídica. Con esto, en los casos en los que surgen defectos morales, se da como consecuencia la exclusión de los hechos sociales de las fuentes del derecho. Agustín nos da una declaración clásica de esta opinión cuando dice que «una ley que no es justa no me parecería que sea una ley» (Augustinus, 2006, 86). Un ejemplo reciente es la tesis propuesta por D. BEYLEVELD y R. BROWNSWORDEN la cual «las normas inmorales no son jurídicamente válidas» (BEYLEVELD y BROWNSWORD, 2001, 76).

La contraparte radical del no-positivismo excluyente es el no-positivismo súperincluyente. El no-positivismo súper-incluyente va hacia el otro extremo, es decir, esta corriente teórica sostiene que la validez jurídica no se ve afectada de ninguna manera por cualquier defecto moral. A primera vista, esta parece ser una versión del positivismo, y no del no-positivismo (WALDRON, 1996, 1566). Esta primera impresión, sin embargo, es reconocida como engañosa, tan pronto como se ve que existen dos tipos diferentes de conexión entre el derecho y la moral: una clasificatoria y otra calificatoria (AleXY, 2002a, 26). Estos dos tipos de conexión se distinguen entre sí a partir de los efectos derivados de los defectos morales. El efecto de una conexión clasificatoria es la pérdida de validez jurídica. Por el contrario, el efecto de una conexión calificatoria es la deficiencia jurídica, el cual no llega sin embargo, al punto de socavar la validez jurídica, pero sí crea una obligación legal o, al menos, un empoderamiento por parte de las cortes de apelación para anular las sentencias injustas expedidas por los tribunales inferiores. La conexión del postulado de «sumisión incondicional» al derecho positivo de I. KANT (KANT, 1996, 506) con la idea de una necesaria dominación del derecho nopositivo sobre el derecho positivo puede leerse como una versión del no-positivismo súper-incluyente (AlEXY, 2008b, 289; AleXY, 2010, 176). Lo mismo es cierto para la tesis de TOMÁs DE AQuino que enuncia que una ley tiránica es la ley, pero «no ley simpliciter ${ }^{1} \gg$ (TOMÁs DE AQUinO, 1962, 947) o, como J. FinNiS señala «no es ley en el sentido focal del término «ley» (FINNIS, 1980, 364).

La tercera versión del no-positivismo, es la del no-positivismo incluyente, la cual se encuentra entre los extremos del no-positivismo excluyente y el no-positivismo súperincluyente. Los planteamientos del no-positivismo incluyente se circunscriben a una

${ }^{1}$ Se refiere a la ley en sentido central o pleno, es decir, la ley justa y adecuadamente promulgada, ver Tomás de Aquino, «Summa Theologiae» I-II q. 92, a. 1 ad 4: «Lex tirannica cum not sit simpliciter lex (Ö). Nota del Traductor. 
posición intermedia de modo que se centran en decir acerca de los defectos morales que: ni siempre socavan la validez jurídica — postura representada por el no-positivismo excluyente, ni que nunca lo hacen, según lo expone el no-positivismo súperincluyente- - . En consecuencia, se afirma que los defectos morales socavan la validez jurídica sólo bajo ciertas condiciones.

El no-positivismo incluyente está dado en su expresión más destacada en la fórmula de RADBRUCH, la cual en su forma más comprimida, es la siguiente: la injusticia extrema no es derecho (RADBRUCH, 2006, 7; Alexy, 2008a, 427-428). De acuerdo con esta fórmula, los defectos morales socavan la validez jurídica si y sólo si el umbral de la extrema injusticia se transgrede. La injusticia por debajo de este umbral se considera parte del concepto de derecho, se acepta como derecho válido pero defectuoso. Esto significa que el no-positivismo incluyente abarca un grado considerable de positividad, es decir, entraña un compromiso con aquello que ha sido expedido autoritativamente y es socialmente eficaz. El no-positivismo del no-positivismo incluyente consiste, primero, en el establecimiento de una frontera exterior del derecho y, en segundo lugar, en la calificación del derecho inmoral o injusto no sólo, como moralmente sino también como jurídicamente defectuoso. Las consecuencias prácticas del establecimiento de una frontera exterior se hacen evidentes cuando se observa la aplicación de la fórmula de RADBRUCH por los Tribunales Alemanes después de la derrota del Nacional Socialismo en 1945, y tras el colapso de la República Democrática Alemana en 1989 (ALEXY, 2008a, 428-432). Una consecuencia práctica de calificar al derecho inmoral o injusto como defectuoso no sólo desde el punto de vista moral, sino también desde el punto de vista jurídico es que los tribunales de apelación adquieren la facultad de anular las sentencias injustas de los jueces inferiores debido a su deficiencia jurídica.

\section{EL ARGUMENTO DE LA CORRECCIÓN}

La existencia de dos formas de positivismo y tres de no-positivismo muestra que el debate entre el positivismo y el no-positivismo se refiere más que a una simple disputa entre dos posiciones monolíticas, a menudo presentada como la oposición entre el «positivismo jurídico» y el «derecho natural». Pero, eso no es todo. Las cosas se complican aún más, tan pronto como se tiene en cuenta el hecho de que no sólo el positivismo así como el no-positivismo son complejos en sí mismos, sino que la complejidad se manifiesta también en la estructura de los argumentos a favor y en contra de cada una de estas corrientes teóricas. El punto arquimédico de esta estructura es el argumento de la corrección. Todos los demás argumentos giran en torno a este punto.

$\mathrm{El}$ argumento de la corrección enuncia que tanto las normas jurídicas individuales así como las decisiones judiciales individuales, al igual que los sistemas jurídicos como un todo necesariamente formulan una pretensión de corrección. R. DwORKIN ha objetado la cuestión acerca de si los operadores jurídicos al elevar o formular alguna pretensión es más una cuestión de hecho que una cuestión de necesidad (DwORKIN, 2006, 200). Esta objeción puede ser rechazada si es posible demostrar que la pretensión de corrección está necesariamente implícita en el derecho, independientemente de las intenciones de sus operadores. Aquí la idea es mostrar que la negación explícita de la pretensión de corrección conduce a una contradicción (ALEXY, 2002a, 35-39). 
Un ejemplo es un primer artículo ficticio de una Constitución que se leería así: «X es una república soberana, federal e injusta». Este artículo es de alguna manera absurdo. El absurdo surge de una contradicción entre lo que se pretende implícitamente con la expedición de una constitución, es decir, lo justo, y lo que se ha declarado explícitamente, esto es, lo injusto. Ahora bien, la justicia cuenta como un caso especial de la corrección, y por justicia debe entenderse que no es otra cosa que la corrección en la distribución y la compensación. Por lo tanto, la contradicción en nuestro ejemplo no es sólo una contradicción con respecto a la dicotomía de lo justo e injusto sino también una contradicción con respecto a la dicotomía de lo correcto e incorrecto.

Es más, en el ejemplo antes mencionado, la contradicción que existe entre lo explícito y lo implícito es necesaria, y podría evitarse si se abandona la pretensión implícita, pero hacerlo representaría la transición de un sistema jurídico a meras relaciones de poder al desnudo (AlEXY, 1998, 213-214). Así, nuestro ejemplo muestra que el derecho y la pretensión de corrección no están solamente conectados por razones contingentes y prudenciales, sino también —y esto va bastante más lejos- por razones necesarias por naturaleza. Esta conexión no está confinada de ninguna manera a actos fundamentales como el de expedir una constitución, ya que está presente en cada parte del sistema jurídico, lo cual puede ser ilustrado por el absurdo encontrado en decisiones como la siguiente: El acusado es condenado a cadena perpetua, en virtud de una incorrecta interpretación de la legislación vigente.

Con el fin de establecer una conexión necesaria entre el derecho y la moral, no es suficiente que la pretensión de corrección sea necesariamente elevada por el derecho. Más allá de esto, es necesario que su contenido necesariamente se refiera a la moral.

La pretensión de corrección del derecho no se referiría necesariamente a la moral si fuera posible que se circunscribiera exclusivamente a hechos sociales, es decir, a lo que ha sido autoritativamente expedido y es socialmente eficaz. Una pretensión con este contenido contaría como una pretensión puramente positivista de corrección. Los casos difíciles, sin embargo, ponen de manifiesto que la interpretación positivista del argumento de la corrección da lugar a serios problemas. Los casos difíciles se producen cuando el derecho positivo - entendido como las razones autoritativas o basadas en fuentes de validez jurídica- permite más de una decisión. La decisión a tomarse en tal esfera «abierta» es una decisión sobre una cuestión normativa que no puede basarse en normas de derecho positivo, pues si pudiera basarse en tales normas, no sería una decisión de esfera «abierta». Si no se basa en alguna norma en absoluto, sería una decisión meramente arbitraria, una decisión que estaría en contradicción con la pretensión de corrección, por ello debe basarse en otros criterios normativos. Las decisiones jurídicas regularmente versan sobre cuestiones de distribución y compensación. Las cuestiones acerca de la correcta distribución y compensación son cuestiones de justicia y las cuestiones de justicia son, sin embargo, cuestiones morales. De esta forma, la textura abierta del derecho, tomada junto con la naturaleza de las cuestiones jurídicas, implica que la pretensión de corrección formulada en la toma de decisiones jurídicas necesariamente se refiere no sólo a razones autoritativas o basadas en fuentes de validez jurídica, sino también a razones morales. Esto implica que la pretensión de corrección necesariamente planteada en el derecho conduce a la necesaria inclusión de la moral en el concepto de derecho (ALEXY, 2007, 49-50). 


\section{LA NATURALEZA DUAL DEL DERECHO}

H. KELSEN ha argumentado en contra del no-positivismo que presupone «una moral absoluta, es decir, una moral que sea válida en todas partes y en todo momento» (KELSEN, 1967, 68 trans. alt). KeLSEN prosigue señalando que no existe tal moralidad absoluta, postura que puede enmarcarse bajo el nombre de «el argumento del relativismo» (ALEXY, 2002a, 53-55). La cuestión de si la objeción de Kelsen basada en el relativismo es convincente, es una cuestión de interpretación. Si se entiende dicha objeción en el sentido de no existir ninguna instancia en la cual una sola respuesta correcta desde el punto de vista moral puede darse, entonces la objeción falla, pues hay casos de grave interferencia en los derechos humanos en los que sólo una respuesta moral es correcta o verdadera, específicamente aquellos en los que tal injerencia viola los derechos humanos y es, por esa razón, que son moralmente incorrectos (ALEXY, 2012, 8-13). La existencia de este tipo casos es suficiente como base epistemológica o meta-ética del no-positivismo. Sin embargo, si se interpreta la objeción de Kelsen en el entendido de la existencia de un número de casos — un número considerable - en los que es posible un «desacuerdo razonable» (RAwLS, 1993, 55) acerca de lo que es moralmente correcto o incorrecto, entonces el argumento de KELSEN está basado en una tesis epistemológica o meta-ética correcta, pero, y este es el punto, ya no sería suficiente como argumento en contra del no-positivismo. El no-positivismo es compatible con el desacuerdo razonable si es posible llegar a una aproximación a la verdad o corrección en el discurso y si hay al menos algunos casos en los que sólo es posible una única respuesta moral correcta, en la cual el desacuerdo razonable no es posible. Una aproximación a la verdad o corrección es posible porque el discurso práctico racional es posible, y hay casos en los que sólo una respuesta moral es posible ya que otros resultados serían discursivamente imposibles — como, por ejemplo- el estatus jurídico de un esclavo o la abolición de la libertad religiosa (ALEXY, 1989, 187-208).

La existencia de un desacuerdo razonable significa que hay un número considerable de problemas sociales, que el argumento moral no puede resolver por sí solo, a lo cual se podría llamar el «problema del conocimiento práctico». Este problema sólo puede resolverse a través de los procedimientos legalmente regulados que garantizan una decisión. Este es el paso de la moral al derecho positivo, como ha sido descrito, por ejemplo, por KANT (KANT, 1996, 456). Es más, el problema del conocimiento práctico no es el único problema que puede resolverse por medio del derecho positivo. Un segundo problema es el problema de la coerción, referido a si es posible violar la ley sin correr ningún riesgo, y si algunos toman ventaja de esta posibilidad, entonces el cumplimiento de las normas ya no está asegurado. En resumen, los procedimientos que garantizan el cumplimiento de la ley son necesarios. Adicionalmente, existe el problema de la organización. Una sociedad moderna puede ser eficazmente organizada sólo a través del derecho positivo.

La necesidad de resolver estos tres problemas y, por tanto, la necesidad de la positividad, es decir, de la expedición autoritativa y de la eficacia social, se deriva de la exigencia moral de evitar los costos de la anarquía y la guerra civil y asegurar las ventajas derivadas de la coordinación social y la cooperación. Como razones morales, éstas son parte del contenido de la pretensión de corrección del derecho. Esto implica que la 
pretensión de corrección del derecho qua pretensión de corrección moral comprende necesariamente elementos de positividad. Esto no quiere decir, sin embargo, que la pretensión de corrección comprende únicamente elementos de positividad. Este es el error del positivismo súper-incluyente. La pretensión de corrección sustancial —que es primero y principalmente, una pretensión de justicia - no desaparece una vez que el derecho se ha institucionalizado, ésta vive en el derecho. Por esta razón, hay que distinguir dos etapas o niveles de corrección: la corrección de primero y de segundo orden.

La corrección de primer orden se trata de la justicia como tal. La corrección de segundo orden es más integral, y se refiere tanto a la justicia como a la positividad. La justicia representa la dimensión ideal o crítica del derecho, la positividad su dimensión real, objetiva o institucional. La pretensión de corrección del derecho qua pretensión de segundo orden une la dimensión real y la dimensión ideal. Es una expresión de la naturaleza dual del derecho (ALEXY, 2010, 173-174).

La naturaleza dual del derecho implica que éste comprende necesariamente dos principios: el principio de la justicia y el principio de seguridad jurídica. El principio de seguridad jurídica es un principio formal, que requiere un compromiso con lo que se expide autoritativamente y es socialmente eficaz. El principio de justicia es principio material o sustantivo que exige que la decisión sea moralmente correcta. Estos dos principios - como generalmente ocurre con los principios- pueden colisionar, y de hecho colisionan con frecuencia. Ninguno de los dos en ningún caso podrá suplantar al otro completamente. Por el contrario, la naturaleza dual del derecho exige que puedan ser vistos en una proporción correcta entre sí, y esto, a su vez, sólo se puede lograr por medio de la ponderación. La idea de una frontera exterior del derecho es el resultado de tal ponderación, esto es, de equilibrar los principios de seguridad jurídica y justicia.

\section{FRONTERA EXTERNA}

La fórmula de RADBRUCH, que en su expresión más corta, establece que la injusticia extrema no es derecho, es la expresión clásica de la idea de una frontera externa del derecho (RADBRUCH, 2006, 7; AlEXY, 2008a, 428). Esta fórmula representa, primero y ante todo, un rechazo a la tesis positivista según la cual «cualquier contenido puede ser derecho» (KELSEN, 1967, 198). KELSEN ilustra esta tesis con la siguiente afirmación: «De acuerdo con el derecho de los estados totalitarios, el Gobierno está facultado para confinar en campos de concentración a las personas de convicciones, religión o raza indeseadas y puede obligarlas a hacer cualquier clase de trabajo, el que sea, incluso puede matarlas» (KELSEN, 1967, 40, trad. alterado). Matar personas en campos de concentración por razones tales como el rechazo a sus convicciones, la religión o la raza es un claro caso de injusticia extrema. Por lo tanto, de acuerdo con la fórmula de RADBRUCH, las normas que permiten a las autoridades legales hacer tales cosas, no pueden ser derecho válido. Desde el punto de vista positivista, la situación es diferente. Si esas normas se han expedido autoritativamente y son socialmente eficaces, son derecho vigente. Esto también es cierto en el positivismo incluyente, en los casos en los que el derecho positivo no se refiere exactamente, en una manera socialmente eficaz a los principios morales que excluyen el asesinato. Desde el punto de vista no-positivista, 
todo depende del equilibrio entre los principios de seguridad jurídica y justicia. El principio de seguridad jurídica sostiene que la norma, en el ejemplo de KELSEN, es jurídicamente válida, el principio de justicia conduce al resultado opuesto. La determinación del equilibrio depende esencialmente de la intensidad de la interferencia en cada uno de los principios en colisión (ALEXY, 2002b, 102). No concebir la norma en cuestión como inválida, plantearía una interferencia muy intensa con el principio de justicia, principio que comprende los derechos humanos, dicha interferencia en el ejemplo de KELSEN es extrema. Por otro lado, la pérdida de seguridad jurídica si se llegara a concebir la norma como inválida sería muy limitada, pues incluso en los estados totalitarios un gran número de normas no superan el umbral de la extrema injusticia. De este modo, se determina el resultado del equilibrio, y este resultado refleja precisamente la fórmula RADBRUCH, es decir, el no-positivismo incluyente tal como se define por dicha fórmula. Desde esta perspectiva, tanto el no-positivismo súper-incluyente así como el no-positivismo excluyente no son acertados. El no-positivismo súper-incluyente falla, ya que da muy poco peso a la justicia, y el no-positivismo excluyente falla también, ya que da muy poca importancia a la seguridad jurídica.

\section{PARTICIPANTES Y OBSERVADORES}

Esta justificación de la fórmula de RADBRUCH, podría objetarse al considerar que tiene puntos débiles en su fundamento ya que se basa en argumentos normativos, específicamente, los principios de justicia y seguridad jurídica. La cuestión de la naturaleza del derecho, la objeción continúa, se refiere a lo que el derecho es, y lo que una cosa es no se puede establecer por medio de argumentos normativos (RAZ, 1996, 7; MARMOR, 2005, 778).

Para responder a esta objeción, la distinción entre la perspectiva del observador y la perspectiva del participante es fundamental (ALEXY, 2002a, 25). Un observador plantea preguntas y aduce argumentos, en nombre de una posición que refleja la forma en que las cuestiones jurídicas son de hecho decididas en un sistema jurídico, mientras que el participante plantea preguntas y aporta argumentos con la vista puesta en lo que él considera es la respuesta correcta a la cuestión jurídica en cuestión. La perspectiva del observador se define por la pregunta «¿Cómo se toman las decisiones jurídicas realmente?» La perspectiva del participante se define en cambio por la pregunta «¿Cuál es la respuesta jurídica correcta?».

El positivismo refleja bastante bien la perspectiva del observador (AleXY, 2002a, 27-35), desde la cual, lo que el derecho es depende exclusivamente de lo que se ha expedido de hecho y es socialmente eficaz. Por el contrario, la perspectiva del participante presupone el no-positivismo. Para un participante el derecho es lo que se ha decidido correctamente, y esto depende, sin embargo, no sólo de hechos sociales, sino también de razones morales. De esta forma, los argumentos normativos toman su camino en lo que el derecho es, tan pronto como la perspectiva del participante es asumida. Es más, este punto de vista debe ser aceptado porque el derecho es posible sin observadores, pero no sin participantes.

(Traducción de María Claudia Quimbayo Duarte) 


\section{BIBLIOGRAFÍA}

Alexy, R. [1978], 1989: A theory of legal argumentation, en R. AdLeR y N. MacCormick (trads.), Oxford: Clarendon Press.

- 1998: Law and correctness. Current Legal Problems, 51, 205-221.

- [1992], 2002a: The argument from injustice. A reply to legal positivism, en B. LITSCHEWSKI Paulson y S. L. Paulson (trads.), Oxford: Clarendon Press.

- [1985], 2002b: A theory of constitutional rights, en J. Rivers (trad.), Oxford: Oxford University Press.

- 2007: «An Answer to Joseph Raz», en G. PAVlakos (ed.), Law, rights and discourse. The legal philosophy of Robert Alexy, 37-65. Oxford: Hart Publishing.

- 2008a: «A defence of Radbruch's formula», en M. D. A. FREEMAN (ed.), Lloyd's introduction to jurisprudence, 426-43, London: Sweet \& Maxwell and Thomson Reuters.

- 2008b: «On the concept and the nature of law», Ratio Juris, 21, 281-99.

- 2010: «The dual nature of law», Ratio Juris, 23, 167-82.

— 2012: «Law, morality, and the existence of human rights», Ratio Juris, 25, 2-14.

Aquinas, T., 1962: Summa theologiae, Turin: Paoline.

Augustinus, 2006: De liberoarbitrio, en J. Brachtendorf y V. H. Decroll (eds.), Paderborn: Schöningh.

Beyleveld, D., y Brownsword, R., 2001: Human dignity in bioethics and biolaw, Oxford: Oxford University Press.

Coleman, J., 1996: «Authority and reason», en R. P. GeORGE (ed.), The autonomy of law. Essays on legal positivism, 287-319, Oxford: Clarendon Press.

Dworkin, R., 2006: Justice in robes, Cambridge, MA: Harvard University Press.

FINNIS, J., 1980: Natural law and natural rights, Oxford: Clarendon Press.

Kant, I. [1797], 1996: «The metaphysics of morals», en I. Kant, Practical philosopby, M. J. Gregor (trad. y ed.), 353-603, Cambridge: Cambridge University Press.

Kelsen, H. [1960], 1967: Pure theory of law, M. KNight (trad.), Berkeley: University of California Press.

Marmor, A., 2005: «Debate», Anales de la cátedra Francisco Suárez, 39, 769-93.

RAdBRUCH, G. [1946], 2006: «Statutory lawlessness and supra-statutory law», B. LITSCHEWSKI Paulson y S. L. Paulson (trads.), Oxford Journal of Legal Studies, 26, 1-11.

Rawls, J., 1993: Political liberalism, New York: Columbia University Press.

RAz, J., 1996: «On the nature of law», Archives for Philosophy of Law and Social Philosophy, 82, $1-25$.

- 2009: The authority of law (2. ${ }^{a}$ ed.), Oxford: Oxford University Press.

WaLdRON, J., 1996: «Kant's legal positivism», Harvard Law Review, 109, 1535-1566.

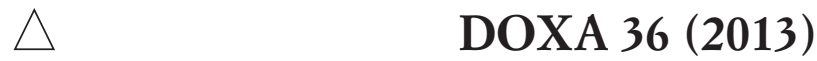

